\title{
Non-vertebral Veillonella species septicemia and osteomyelitis in a patient with diabetes: a case report and review of the literature
}

\author{
Fawzia Eida Al-Otaibi and Maha Mohammed Al-Mohizea
}

\begin{abstract}
Introduction: Veillonella is a nonfermentative, strictly anaerobic, Gram-negative coccus that forms part of the human gastrointestinal tract, mouth and vaginal flora. Like other anaerobic infection, Veillonella species usually are involved in polymicrobial processes, which make it difficult to determine their pathogenic role. Isolation of a clinically significant Veillonella species is rare and $V$. parvula is the most common one reported to cause infection in humans. The most frequently reported infection caused by $V$. parvula is osteomyelitis, almost always in association with bacteremia.

Case presentation: Here, we describe a rare case of nonvertebral osteomyelitis and septicemia caused by Veillonella species in a 49-year-old Saudi man with diabetes. Initial treatment with ciprofloxacin was associated with treatment failure and poor response. Identification of the organism was essential for the selection of appropriate treatment. There have been only seven previous reports of Veillonella vertebral osteomyelitis and one report of V. parvula foot osteomyelitis with sepsis in the literature. This is the second case of Veillonella nonvertebral osteomyelitis associated with septicemia reported to date.

Conclusions: Veillonella species should be considered a true pathogen in diabetic patients with osteomyelitis and those with underlying immune suppression, particularly if the organism is isolated from blood. The isolation of those obligate anaerobes from blood may signal the presence of severe underlying disease and the probable need for timely surgical intervention.
\end{abstract}

Keywords: Diabetic foot, Osteomyelitis, Veillonella bacteremia

\section{Introduction}

Veillonella is a nonfermentative, small, nonmotile, strictly anaerobic, Gram-negative cocci that form part of the normal flora of the oral, respiratory, intestinal, and female genital tracts [1]. They are usually recovered as part of a polymicrobial infection and they are often regarded as a contaminant. However, they have been isolated in pure culture from various sterile body sites such as sinuses, lungs, liver, central nervous system, heart, and bone. Veillonella parvula is the most common clinically significant species and is infrequently grow from blood cultures. We report here a case of Veillonella species bacteremia associated with osteomyelitis in which pure Veillonella species

\footnotetext{
* Correspondence: ofawzia@ksu.edu.sa

King Khalid University Hospital, King Saud University, PO: 2925, Riyadh 11461, Saudi Arabia
}

was isolated from blood culture. This is the second reported case of Veillonella nonvertebral osteomyelitis, which illustrates the pathogenic potential of this organism in causing invasive infection in diabetic patients.

\section{Case presentation}

A 49-year-old Saudi man, a known case of insulindependent diabetes mellitus (IDDM), hypertension and ischemic heart disease, presented to the emergency department with a three-day history of fever, chills and ankle pain after trauma to his left foot. He reported a nonhealing ulcer on his left heel following a hot surface injury 20 days prior to his presentation. On examination, our patient looked ill and septic, with minimal ambulation. His temperature was $38.9^{\circ} \mathrm{C}$ and his blood pressure and pulse were $140 / 90 \mathrm{mmHg}$ and 108 beats/ minutes respectively. A lower limb examination revealed 
bilateral decrease of sensation and a foot ulcer on his left heel. Careful examination of his left foot showed a $7 \times 5 \mathrm{~cm}$ deep ulcer on the lateral side that looked badly infected with change of skin color and profuse pus discharge. Our patient was seen by the orthopedic surgeon and an urgent extensive wound debridement involving the bone was undertaken. Blood, pus and bone tissue specimens were collected and sent to the microbiology laboratory for culture and sensitivity testing. A laboratory examination showed a white cell count (WBC) of $29 \times 10^{9} \mathrm{~g} / \mathrm{L}$ with $90 \%$ neutrophils, hemoglobin of $6.9 \mathrm{~g} / \mathrm{L}$, and an erythrocyte sedimentation rate (ESR) of $97 \mathrm{~mm} / \mathrm{h}$. His serum creatinine
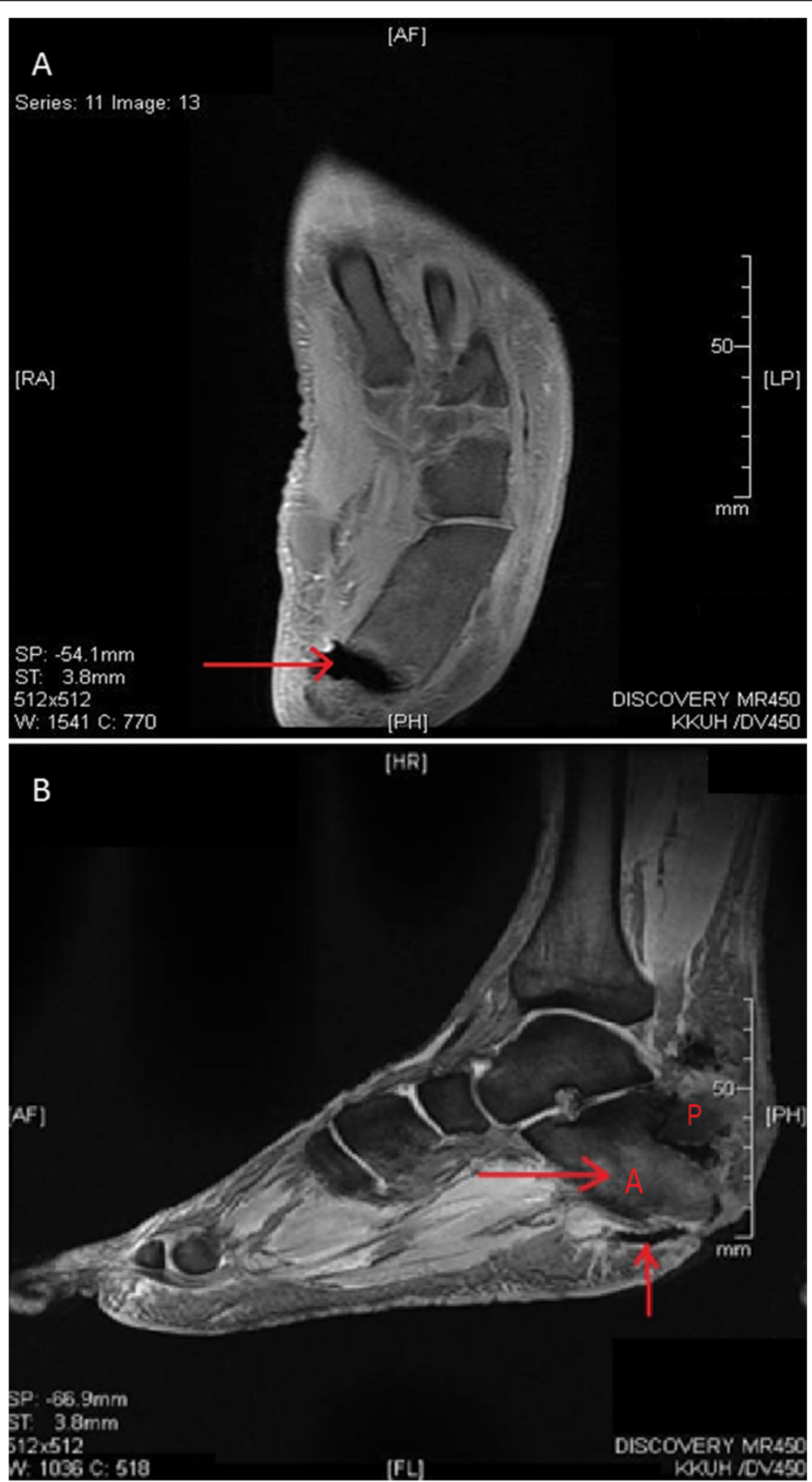

Figure 1 Left foot magnetic resonance imaging. (A) A left foot MRI scan revealed a fracture in the calcaneus. (B) The calcaneus bone is separated into two segments ((A) and (P)) with evidence of anterior segment osteomyelitis and a deep, infected ulcer (arrows). 
was $123 \mathrm{umol} / \mathrm{L}$, and urea $\mathrm{mmol} / \mathrm{L}$ 8.9. His liver function test results were within normal limits except for serum alkaline phosphatase (ALP), which was raised (785IU/L). A left foot X-ray revealed soft tissue swelling, gas formation and fracture of the calcaneus bone. A left foot magnetic resonance imaging (MRI) scan revealed a calcaneus fracture with high suspicion of calcaneus anterior fragment osteomyelitis (Figure 1). An MRI scan of his right foot revealed mild neuropathic arthropathy (Charcot joint) with no evidence of osteomyelitis. Our patient was started empirically on intravenous ciprofloxacin 400mg twice daily, and intravenous clindamycin 600mg eight hourly. During the first three days after starting treatment, our patient showed no clinical improvement and he continued to run a low-grade fever. A pus culture grew Escherichia coli sensitive to ciprofloxacin, imipenem, meropenem, gentamicin and Enterococcus avium sensitive to ampicillin. A bone and tissue biopsy grew Morganella morganii sensitive to gentamicin, imipenem and resistant to ciprofloxacin, ampicillin, amoxicillin-clavulanic acid, piperacillin/tazobactam and cotrimoxazole. No growth occurred on the anaerobic plates despite prolonged incubation. Based on culture results, the initial antibiotics were suspended and our patient was started on imipenem 500mg intravenously (IV) six hourly, vancomycin $1 \mathrm{~g}$ IV 12 hourly, and colistin 2 million units IV eight hourly. The blood culture obtained before treatment initiation, which was inoculated in an anaerobic bottle (Bactec Lytic/10, Anaerobic/F, Beckton Dickinson, Franklin Lakes, NJ, USA) and processed by an automated blood culture system (Bactec FX, Beckton Dickinson), grew very scanty Gramnegative cocci within 18 hours shown on Gram stain. The blood then was subcultured on Trypticase soy agar with sheep blood (BBL Microbiology Systems, Cockeysville, $\mathrm{MD}$, USA) and incubated at $35^{\circ} \mathrm{C}$ in $5 \% \mathrm{CO} 2$, in an anaerobic GasPak $^{\mathrm{Tm}}$ jar (BBL Microbiology Systems). After 48 hours of anaerobic incubation, the blood agar grew slowgrowing tiny colonies (Figure 2) that showed Gramnegative cocci on Gram stain (Figure 3). The organism was identified by the Vitek 2 automated system (bioMérieux, Marcy-l'Étoile, France), as Veillonella species with a $99 \%$ probability rate. It was sensitive to imipenem $(1.5 \mu \mathrm{g} / \mathrm{ml})$, clindamycin $(0.047 \mu \mathrm{g} / \mathrm{ml})$, ceftriaxone $(8 \mu \mathrm{g} / \mathrm{ml})$ and colistin, and resistant to penicillin $(>32 \mu \mathrm{g} / \mathrm{ml})$, vancomycin, erythromycin and metronidazole. Further identification to species level and genotyping was unable to be performed, as the subcultured isolates failed to grow and the significant time delay before attempting to reisolate the organisms from the blood culture bottle rendered them nonviable. Over the subsequent five days, our patient's condition worsened with a high temperature $\left(38.9^{\circ} \mathrm{C}\right)$, rigors, a high WBC and ESR count. The wound was reevaluated by the surgical team and a decision of above-knee amputation was taken. Colistin and vancomycin

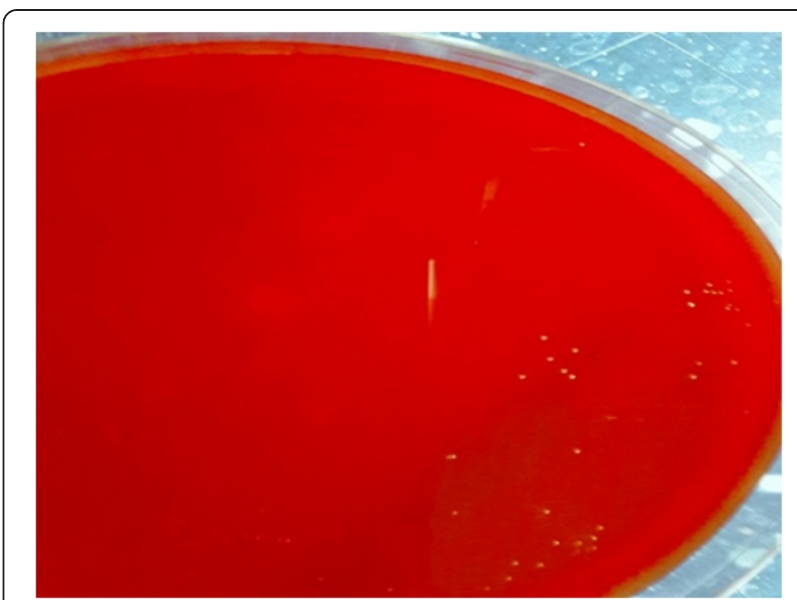

Figure 2 Blood agar plate after $\mathbf{4 8}$ hours of anaerobic incubation showing tiny colonies of Veillonella organism.

were discontinued and imipenem was continued for four weeks. On follow-up, our patient showed gradual improvement of his condition and was discharged.

\section{Discussion}

Veillonella species are rare causes of serious infections such as meningitis [2], endocarditis [3], obstructive pneumonitis [4], prosthetic joint infection [5], and bacteremia [6]. Bacteremia has been reported almost always in association with an underlying infection such as osteomyelitis. There have been seven previous reports of vertebral osteomyelitis [7-13] and one report of foot cellulitis and osteomyelitis in a middle-aged woman associated with sepsis caused by $V$. parvula [14] (Table 1). Our report is considered to be the second report of Veillonella nonvertebral osteomyelitis associated with bacteremia. Borchardt [14] reported the first case of nonvertebral osteomyelitis in which a 54-year-old Indian diabetic woman developed foot cellulitis and toe osteomyelitis after attempting to

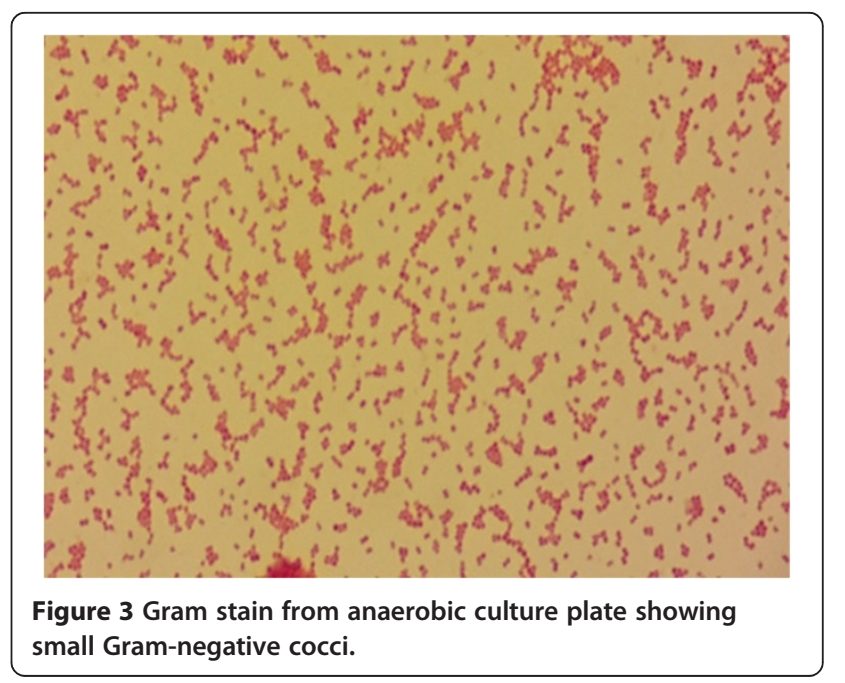


Table 1 Previously reported cases of osteomyelitis caused by Veillonella

\begin{tabular}{|c|c|c|c|c|c|c|}
\hline \multirow{2}{*}{$\begin{array}{l}\text { Number of patients } \\
\text { (reference) }\end{array}$} & \multirow[t]{2}{*}{ Age (year)/sex } & \multicolumn{2}{|c|}{ Culture result } & \multirow[t]{2}{*}{ Underlying disease/risk factors } & \multirow[t]{2}{*}{ Type of bone/soft tissue } & \multirow[t]{2}{*}{ Antibiotic therapy } \\
\hline & & Blood & Bone/disc & & & \\
\hline 1. (This study) & $49 / M$ & $\begin{array}{l}\text { Veillonella } \\
\text { sp. }\end{array}$ & NR & Diabetes mellitus and diabetic foot & Foot bone (Calcenous) & Imipenem \\
\hline 2. Marriott [7] & $55 / \mathrm{M}$ & V. parvula & V. parvula & Colonoscopy & Lumbar spine discitis & Ceftriaxone \\
\hline 3. Singh $[8]$ & $61 / F$ & V. parvula & V. parvula & Sjogren's syndrome, xerostomia- & Thora columbar spine & Ceftriaxone \\
\hline 4. Bongaerts [9] & $74 / \mathrm{M}$ & NR & V. parvula & None & Spine & Penicillin \\
\hline 5. Hidalgo [10] & 70/M & NR & V. parvula & None & Spine & US \\
\hline 6. Isner-Horobeti [11] & $27 / \mathrm{M}$ & NR & Veillonella sp. & None & Lumbar spondylodiscitis & Amoxicillin \\
\hline 7. Kishen [12] & $76 / F$ & $\begin{array}{l}\text { Veillonella } \\
\text { sp. }\end{array}$ & Veillonella sp. & None & $\begin{array}{l}\text { Lumbar spondylodiscitis and } \\
\text { paraspinal space abscesses }\end{array}$ & $\begin{array}{l}\text { Spinal surgery + Cefotaxime/ } \\
\text { metronidazole }\end{array}$ \\
\hline 8. Barnhart [13] & $31 / \mathrm{M}$ & NR & Veillonella sp. & $\begin{array}{l}\text { Cervical vertebral fracture/Post cervical } \\
\text { spine fusion }\end{array}$ & $\begin{array}{l}\text { Cervical spine and retropharyngeal } \\
\text { abscess }\end{array}$ & Penicillin $\mathrm{G}+$ abscess drainage \\
\hline 9. Borchardt [14] & $54 / F$ & V. parvula & V. parvula & Diabetes mellitus and foot shaving injury & $\begin{array}{l}\text { Phalanx and metatarsal bone and } \\
\text { cellulitis }\end{array}$ & Excision of infected bone + penicillin \\
\hline
\end{tabular}


shave a callus on her right foot two months before presentation. The patient recovered completely after excision of the phalanx and metatarsal bones along with four weeks of intravenous penicillin treatment. $V$ parvula was isolated from the excised tissue and the blood culture. In five of the previously reported clinical infections, the patients were previously healthy and the portal of entry was not identified. Isner-Horobeti [11] reported on a 27-year-old man with unknown risk factors for infection, who presented with an L4 to L5 spondylodiscitis. The patient was cured after a prolonged course (11 weeks) of intravenous followed by oral penicillin. In another report by Bongaerts [9] V. parvula was isolated from the spine (T12 to L1) of a previously healthy 74-year-old man who was treated with six weeks of intravenous penicillin. Similar cases were reported by Hidalgo [10] and Kishen et al. [12] of Veillonella spondylodiscitis in older patients without any risk factors. The outcome of the patients was favorable after antibiotic treatment alone or combined with surgical management. On the other hand, colonoscopy was considered by Marriott [7] as a possible source of entry of $V$. parvula causing bacteremia and lumbar discitis in a 55-year-old man who had undergone small intestinal and rectal biopsies eight weeks prior to presentation. In another report by Barnhart [13], postoperative Veillonella osteomyelitis of the cervical spine was reported from a 31-year-old man who had suffered a cervical fracture at the level of the fourth and fifth cervical vertebrae two months before presentation. The possible source of entry of the organism was thought to be the esophageal perforation that had occurred during surgery. Veillonella was isolated repeatedly from a retropharyngeal soft tissue abscess, which was drained followed by parenteral penicillin administration for six weeks. The best method for identification of Veillonella strains at the species level requires direct sequencing of the 16S rRNA gene [15]. Conventional phenotypic and biochemical testing does not provide adequate discrimination between species. The pathogenic role of these anaerobes has not been established. However, previously published reports demonstrated their role as a true pathogen associated with fatal overwhelming septicemia [6]. In our case, in which Veillonella species was isolated in pure culture from blood in a patient with signs of severe diabetic wound infection illustrates the pathogenic role of this microorganism. The strictly anaerobic organism failed to grow from the wound probably due to improper sample collection and transport supportive of anaerobic culture. In addition, the presence of other rapidly growing aerobic organisms as part of the polymicrobial infection might interfere with their recovery from wound infection. Veillonella is usually vancomycin, tetracycline, aminoglycosides, and ciprofloxacin resistant and infection typically responds well to therapy with penicillin. Other antimicrobial agents to which the organism is usually susceptible in vitro include cephalosporins, clindamycin, metronidazole, and chloramphenicol. There are no clear treatment recommendations in the literature due to the scarce number of reports on Veillonella as a pathogen associated with invasive infection. However, the previous few reports showed good response to antibiotics such as penicillin, cephalosporins, chloramphenicol, clindamycin and metronidazole [16-20]. Metronidazole was suggested by Warner et al. [16] as an effective drug for the management of serious infections like bacteremia, brain abscess, and meningitis. The isolate from our case showed unusual high minimum inhibitory concentration (MIC) to penicillin $(>32 \mu \mathrm{g} / \mathrm{ml})$. However, in a recent study Veillonella species isolated from the oral cavities of humans demonstrated a high level of resistance to penicillin G (MIC, $2 \mu \mathrm{g} / \mathrm{ml}$ ) [21] and reduced susceptibility to ampicillin or amoxicillin. Reports on the management of infections caused by Veillonella isolates demonstrating high MIC to penicillin is lacking due to limited published studies on the susceptibility of Veillonella species to different antimicrobial agents, particularly penicillin, and their clinical effectiveness.

\section{Conclusion}

Veillonella species should be considered a true pathogen in diabetic patients with osteomyelitis and those with underlying immune suppression, particularly if the organism is isolated from blood. The isolation of those obligate anaerobes from blood may signal the presence of severe underlying disease and the probable need for timely surgical intervention.

\section{Consent}

Written informed consent was obtained from the patient for publication of this case report and any accompanying images. A copy of the written consent is available for review by the Editor-in-Chief of this journal.

\section{Abbreviations}

ALP: alkaline phosphatase; ESR: erythrocyte sedimentation rate; IDDM: insulin-dependent diabetes mellitus; IV: intravenous;

MIC: minimum inhibitory concentration; MRI: magnetic resonance imaging; NR: not reported; US: unspecified; USA: United States of America; WBC: white cell count.

\section{Competing interests}

The authors declare that they have no competing interests.

\section{Authors' contributions}

FEA and MMA were involved in collecting the data and writing and critically reviewing the manuscript. Both authors read and approved the final manuscript.

\section{Acknowledgments}

The authors would like to thank the medical report department and the library personal for their help in providing access to get information related our case.

Received: 30 April 2014 Accepted: 27 August 2014

Published: 12 November 2014 
1. Delwiche EA, Pestka JJ, Tortello ML: The Veillonellae: gram negative cocci with a unique physiology. Annu Rev Microbio/ 1985, 39:175-193.

2. Nukina S, Hibi A, Mishida K: Bacterial meningitis caused by Veillonella parvula. Acta Paediatr Jpn 1989, 31:609-614.

3. Loughrey AC, Chew EW: Endocarditis caused by Veillonella dispar. J Infect 1990, 21:319-321.

4. Liaw YS, Yang PC, Wu ZG, Yu CJ, Chang DB, Lee LN, Kuo SH, Luh KT: The bacteriology of obstructive pneumonitis. A prospective study using ultrasound-guided transthoracic needle aspiration. Am J Resp Crit Care Med 1994, 149:1648-1653.

5. Marchandin H, Teyssier C, Carriere C, Canovas F, Daras H, Jumas-Bilak E: Prosthetic joint infection due to Veillonella dispar. Eur J Clin Microbiol Infect Dis 2001, 20:340-342

6. Liu JW, Wu JJ, Wang LR, Teng LJ, Huang TC: Two fatal cases of Veillonella bacteraemia. Eur J Clin Microbiol Dis 1998, 17:62-64.

7. Marriott D, Stark D, Harkness J: Veillonella parvula discitis and secondary bacteremia: a rare infection complicating endoscopy and colonoscopy? J Clin Microbiol 2007, 45:672-674. Epub 2006 Nov 15.

8. Singh N, Yu VL: Osteomyelitis due to Veillonella parvula. Case report and review. Clin Infect Dis 1992, 14:361-363.

9. Bongaerts GPA, Schreurs BW, Verduyn Lunel F, Lemmens JAM, Pruszynski M, Merkx MAW: Was isolation of Veillonella from spinal osteomyelitis possible due to poor tissue perfusion? Med Hypoth 2004, 63:659-661.

10. Hidalgo C, Piedrola G, Guzman M, Jimenenez-Alfonso JF: Backache in a 70-year old man. Enferm Infecc Microbiol Clin 2000, 18:241-242.

11. Isner-Horobeti M, Lecocq J, Dupeyron A, De Martino SJ, Froehlig P Vautravers P: Veillonella discitis. A case report. Joint Bone Spine 2005, 73:113-115.

12. Kishen TJ, Lindstrom ST, Etherington G, Diwan AD: Veillonella spondylodiscitis in a healthy 76-year-old lady. Eur Spine J 2012, Suppl 4:413-417.

13. Barnhart RA, Weitekamp MR, Aber RC: Osteomyelitis caused by Veillonella. Am J Med 1983, 74:902-904.

14. Borchardt KA, Baker M, Gelber R: Veillonella parvula septicemia and osteomyelitis. Ann Intern Med 1977, 86:63-64.

15. Marchandin H, Teyssier C, de Buochberg MS, Jean-Pierre H, Carriere C, Jumas-Bilak E: Intra-chromosomal heterogeneity between the four 16S rRNA gene copies in the genus Veillonella: implications for the phylogeny and taxonomy. Microbiology 2003, 149:1493-1501.

16. Warner JF, Perkins RL, Cordero L: Metronidazole therapy of anaerobic bacteremia, meningitis, and brain abscess. Arch Intern Med 1979, 139:167-169.

17. Rolfe RD, Finegold SM: Comparative in vitro activity of ceftriaxone against anaerobic bacteria. Antimicrob Agents Chemother 1982, 22:338-341.

18. Rolfe RD, Finegold SM: Comparative in vitro activity of new beta-lactam antibiotics against anaerobic bacteria. Antimicrob Agents Chemother 1981 20:600-609.

19. Sutter VL, Finegold SM: Susceptibility of anaerobic bacteria to 23 antimicrobial agents. Antimicrob Agents Chemother 1976, 10:736-752.

20. Martin WJ, Gardner M, Washington JA: In vitro antimicrobial susceptibility of anaerobic bacteria isolated from clinical specimens. Antimicrob Agents Chemother 1972, 1:148-158.

21. Nyfors S, Kononen E, Bryk A, Syrjanen R, Jousimies-Somer H: Age-related frequency of penicillin resistance of oral Veillonella. Diagn Microbiol Infect Dis 2003, 46:279-283.

doi:10.1186/1752-1947-8-365

Cite this article as: Al-Otaibi and Al-Mohizea: Non-vertebral Veillonella species septicemia and osteomyelitis in a patient with diabetes: a case report and review of the literature. Journal of Medical Case Reports $20148: 365$

\section{Submit your next manuscript to BioMed Central and take full advantage of:}

- Convenient online submission

- Thorough peer review

- No space constraints or color figure charges

- Immediate publication on acceptance

- Inclusion in PubMed, CAS, Scopus and Google Scholar

- Research which is freely available for redistribution

Submit your manuscript at www.biomedcentral.com/submit
C Biomed Central 